\title{
Vacuum Particle Creation: Analogy with the Bloch Theory in Solid State Physics
}

\author{
S.A. Smolyansky*1, A.V.Tarakanov ${ }^{1}$, and M. Bonitz ${ }^{* * 2}$ \\ ${ }^{1}$ Saratov State University, RU-410026, Saratov, Russia \\ ${ }^{2}$ Institut für Theoretische Physik und Astrophysik, Christian-Albrechts-Universität zu Kiel, D-24098 Kiel
}

Received 15 April 2009, accepted 30 June 2009

Published online 08 October 2009

Key words Electron-hole excitation, strong laser field, nonperturbative kinetics, nonstationary Zener effect. PACS 05.30.-d;78.40.Fy;12.20.-m

\begin{abstract}
A nonperturbative kinetic description of interband tunneling under the action of a strong electric field (dynamical analogue of the Zener effect) is presented. The developed approach is based on the similarity to the Sauter-Schwinger effect and its dynamical analogue in QED. The kinetic equation for electron-hole quasiparticle excitations is derived on a dynamical basis in the framework of the oscillator representation. Numerical estimates are made for some simple cases of external fields. Detailed comparisons with the method based on the Bloch equations for electron-hole systems interacting with a time dependent electric field are performed. The proposed approach is an alternative to the Bloch theory and does not use the dipole approximation. This leads to predictions which differ from the ones based on the Bloch equations, in particular, concerning the frequency dependence of the absorption coefficient.
\end{abstract}

(c) 2009 WILEY-VCH Verlag GmbH \& Co. KGaA, Weinheim

\section{Introduction}

The fundamental analogy between the Zener mechanism of interband tunneling [1,2] and Schwinger's mechanism of vacuum electron-positron pair creation in a strong electric field has been mentioned repeatedly, see, e.g., [3]. This similarity has been used recently for the investigation of the transport properties of strongly correlated quantum many-body systems [4].

In this work, we develop a nonperturbative kinetic theory of interband electron-hole $(\mathrm{e}-\mathrm{h})$ excitations in dielectrics under the action of a strong electric laser field using the direct analogy to the QED formalism of vacuum pair creation. The proposed method allows us to take into account on a nonperturbative basis both, tunnel and multi-photon processes, accompanying excitation by an arbitrary time-dependent electric field. Conditionally, one can speak about the dynamical Zener effect by analogy with the dynamical Schwinger effect in QED.

The present work is organized as follows. The definition of the mixed e-h states is an essential distinctive element of our approach. We are employing the rich experience of relativistic QED in describing the vacuum particle creation under the action of some external quasi-classical field (the Schwinger mechanism $[5,6]$ ) and also use some naive analogy between the Dirac picture of electron-positron vacuum and some concepts of solid state band theory as a foundation of our approach. In the framework of the two-band model this leads to a secondorder (with respect to time) equation of motion for the single wave function describing both (electron and hole) states (Sect. 2). For simplicity, electron and hole dispersions are assumed to be the same. For a non-interacting e-h system we construct the Lagrange and Hamilton formalisms and define relevant decompositions of the field functions and canonical momentum with respect to the plane waves corresponding to electron and hole. Thus the formalism represents a special form of e-h representation as a coherent e-h pair state. The interaction with an external quasiclassical time-dependent homogeneous electric field is introduced as result of the standard substitution $\mathbf{p} \rightarrow \mathbf{P}=\mathbf{p}-e \mathbf{A}(t)$. It is well known that the introduction of the external electromagnetic field in QED leads to a non-diagonal form of the commutative operators corresponding to physical observables, what makes

* Corresponding author: e-mail: smol@sgu.ru

** e-mail: bonitz@physik.uni-kiel.de 
the physical interpretation of the formalism difficult [6]. The transition to a quasiparticle (QP) representation is achieved by diagonalization of all commutative operators relevant to the complete QP characteristics of the system (e.g., energy, spin, charge). In practice, only the Hamiltonian diagonalization is often employed. Usually, the transition to the QP representation is done by a time-dependent Bogoliubov transformation (e.g., [6]). The holomorphic (oscillator) representation developed during recent years [7] is a more effective tool for this goal compared to the Bogoliubov technique because it easily allows to obtain the diagonal Hamiltonian and to derive the Heisenberg-like equations of motion for creation and annihilation operators, where it also takes into account the mixing of the states with positive and negative energies. These equations provide the basis for a nonperturbative derivation of the kinetic equation (KE) describing the e-h pair creation and annihilation in the presence of an external electric field (Sect. 3). Excitation of e-h currents leads to generation of an internal electric field, which takes part, in its turn, in the e-h creation process. The corresponding Maxwell equation jointly with the KE put together the complete equation system of the back reaction problem. The mathematical structure of this $\mathrm{KE}$ is similar to the corresponding KE in QED [6, 8], on the one hand, and the Bloch equations in the solid state physics [9-11], on the other. The lines of similarity and distinction are discussed in detail in Sect. 4. The suggested formalism is close to the Galitsky- Goreslavsky-Yelesin theory $[12,13]$ and is an alternative to the Bloch approach and is not based on the dipole model of an electron interaction with an electromagnetic field. Sect. 5 is dealing with the linear response of the electron-hole system (optical absorption spectrum and conductivity) to a weak external electric field. Here we observe certain differences with the results from the Bloch theory. The Sect. 6 contains a short summary.

The developed formalism reveals a close similarity to the corresponding QED kinetics of vacuum electronpositron plasma created under the action of a strong electromagnetic field [8]. The experimental prove of this effect is however difficult [14]. Therefore the study of similar effects in the solid state plasma could be useful for simulation and prediction of the corresponding effects in strong laser fields. We limit ourselves below to the collisionless approximation and neglect the interaction between different charge carriers. We use the units $\hbar=c=1$ throughout the paper.

\section{Description of electron-hole pair states}

Let us consider a two-band system with a completely filled lower band and the mirrored electron states in the $\mathrm{v}$ - and c-zones and dispersions, $\varepsilon_{c}=\Delta / 2+\varepsilon(\mathbf{p}), \varepsilon_{v}=-\Delta / 2-\varepsilon(\mathbf{p})$. It is assumed that the energy gap $\Delta$ is space homogeneous and stationary. The dispersion law $\epsilon(\mathbf{p})$ is fixed on the phenomenological level. We disregard all Coulomb effects and treat electrons and holes as quasi-free particles. Two Schrödinger equations can be associated with these dispersions:

$$
(\hat{E}-\hat{H}) \psi=0,
$$

where $\hat{E}=i \partial / \partial t$ and

$$
\psi=\left\|\begin{array}{l}
\psi_{e} \\
\psi_{h}
\end{array}\right\|, \quad \hat{H}=\left\|\begin{array}{cc}
\Delta / 2+\varepsilon(\hat{\mathbf{p}}) & 0 \\
0 & -\Delta / 2-\varepsilon(\hat{\mathbf{p}})
\end{array}\right\|
$$

with $\hat{\mathbf{p}}=-i \nabla$. In general case for arbitrary dispersions, this leads to independent description of electron and holes. This more complicated case is in need of separate analysis. In this section it is assumed that the external electromagnetic fields and interparticle interaction are absent.

On the other hand, we can consider a hole as an antiparticle to the electron. Their states are correlated and allow for a joint description in analogy with QED. This results in the second order dispersion

$$
\left(E-\varepsilon_{e}\right)\left(E-\varepsilon_{h}\right)=[E-\Delta / 2-\varepsilon(\mathbf{p})][E+\Delta / 2+\varepsilon(\mathbf{p})]=0
$$

(the analogous determinant equation is used in the theory of the stationary Zener effect). The corresponding equation of motion for the total wave function which is second order with respect to time,

$$
\left\{\hat{E}^{2}-[\Delta / 2+\varepsilon(\hat{\mathbf{p}})]^{2}\right\} \Psi=0,
$$


where $\Psi(\mathbf{x}, t)$ is now the one-component wave function. Transition from Eq.(1) to Eq.(4) is very important for our aim.

Our next goal is to develop the Lagrange and Hamilton formalisms for this equation Eq.(4). For simplicity, we use here and below the quadratic isotropic dispersion law, $\varepsilon(\hat{\mathbf{p}})=\hat{\mathbf{p}}^{2} / 2 m$. We obtain then $\left(\Psi_{, k}=\partial \Psi / \partial x_{k}, k=\right.$ $1,2,3)$

$$
L[\Psi]=\alpha\left\{\dot{\Psi}^{*} \dot{\Psi}-\frac{\Delta}{2 m} \Psi_{, k}^{*} \Psi, k-\frac{1}{4 m^{2}} \Psi_{, k l}^{*} \Psi, k l-\frac{\Delta^{2}}{4} \Psi^{*} \Psi\right\},
$$

where the dimensional constant $\alpha$ will be determined below. Let us take advantage of the standard definitions of the canonical momentum and the Hamiltonian density

$$
\pi=\frac{\partial L[\Psi]}{\partial \dot{\Psi}}, \quad \pi^{*}=\frac{\partial L[\Psi]}{\partial \dot{\Psi}^{*}}, \quad H=\pi \dot{\Psi}+\pi^{*} \dot{\Psi}^{*}-L[\Psi] .
$$

Using Eq. (5), we obtain $\pi=\alpha \dot{\Psi}^{*}$ and

$$
H=\frac{1}{\alpha} \pi \pi^{*}+\frac{\alpha}{4} \Delta^{2} \Psi^{*} \Psi+\alpha\left[\frac{\Delta}{2 m} \Psi_{, k}^{*} \Psi_{, k}+\frac{1}{4 m^{2}} \Psi_{, k l}^{*} \Psi_{, k l}\right],
$$

so that the Hamiltonian density contains also higher-order space derivatives.

As in QFT, the wave function $\Psi$ now looses the meaning of the state amplitude. The corresponding charge and current densities are $(\mathrm{k}=1,2,3)$

$$
\begin{aligned}
\rho & =i \alpha e\left(\Psi^{*} \dot{\Psi}-\dot{\Psi}^{*} \Psi\right)=i e\left(\Psi^{*} \pi^{*}-\pi \Psi\right), \\
j_{k} & =i e \alpha\left\{\frac { \Delta } { 2 m } \left[\left(\Psi_{, k}^{*} \Psi-\Psi^{*} \Psi, k\right]-\frac{1}{4 m^{2}}\left[\left(\Psi^{*} \Psi_{, k l l}-\Psi_{, k l l}^{*} \Psi+\Psi_{, k l}^{*} \Psi_{, l}-\Psi_{, l}^{*} \Psi, k l\right]\right\},\right.\right.
\end{aligned}
$$

where $e$ is the electron charge. On this stage, parameter $\alpha$ can be fixed as $\alpha=\Delta^{-1}$ proceeding from the specific correspondence principle: at $\Delta \rightarrow \infty$ the derived relations must turn into the ordinary quantum mechanical analogies (the parameter $\Delta / 2$ plays the role of the rest mass in the Einstein relation for energy and momentum; in order to neglect the state with negative energy, one can use the wave function transformation $\Psi=\Phi e^{-i \Delta t / 2}$.

The transition to the momentum representation can be done in analogy with QFT. Let us carry out the decomposition of wave function $\Psi(\mathbf{x}, t)$ into a plane wave basis (below $\mathbf{p}$ is quasi-momentum, $p_{n}=(2 \pi / L) n_{k}, n_{k}=$ $0,1,2, \ldots$, vector $\mathbf{p}$ belongs to the first Brillouin zone, the crystal volume is $V=L^{3}$ )

$$
\Psi(\mathbf{x}, t)=\frac{1}{\sqrt{V}} \sum_{\mathbf{p}} \int d E \tilde{\Psi}(E, \mathbf{p}) e^{-i E t+i \mathbf{p x}}
$$

and take into account the dispersion equation (3) in order to select the two energy bands

$$
\tilde{\Psi}(E, \mathbf{p})=\delta\{[E-\varepsilon(\mathbf{p})-\Delta / 2][E+\varepsilon(\mathbf{p})+\Delta / 2]\} \psi(E, \mathbf{p}) .
$$

Using the textbook relation $\delta[\phi(x)]=\sum_{i}\left\{\left|\phi^{\prime}\left(x_{i}\right)\right|\right\}^{-1} \delta\left(x-x_{i}\right), \quad \phi\left(x_{i}\right)=0$ the decomposition (10) can be written in the form typical for a two-band model (the electron state is a superposition of $c$ and $v$ band contributions)

$$
\Psi(\mathbf{x}, t)=\frac{1}{\sqrt{V}} \sum_{\mathbf{p}} \sqrt{\frac{\Delta}{\Delta+2 \varepsilon(\mathbf{p})}}\left\{a_{c}(\mathbf{p}) e^{-i[\Delta / 2+\varepsilon(\mathbf{p})] t}+a_{v}(-\mathbf{p}) e^{i[\Delta / 2+\varepsilon(\mathbf{p})] t}\right\} e^{i \mathbf{p} \mathbf{x}},
$$

where $a_{c}(\mathbf{p})$ and $a_{v}(-\mathbf{p})$ are the electron and hole amplitudes in momentum representation. In Eq.(12) we redefined the amplitudes $[\Delta(\Delta+2 \varepsilon)]^{-1 / 2} a_{c, v} \rightarrow a_{c, v}$ in order to guarantee the correct physical interpretation of the observable quantities. The canonical momentum in the same representation (12) has the form

$$
\pi(\mathbf{x}, t)=\frac{i}{2} \frac{1}{\sqrt{V}} \sum_{\mathbf{p}} \sqrt{\frac{\Delta+2 \varepsilon(\mathbf{p})}{\Delta}}\left\{a_{c}^{\dagger}(\mathbf{p}) e^{i[\Delta / 2+\varepsilon(\mathbf{p})] t}-a_{v}^{\dagger}(-\mathbf{p}) e^{-i[\Delta / 2+\varepsilon(\mathbf{p})] t}\right\} e^{-i \mathbf{p x}} .
$$


The transition to the second quantization representation is based on the standard replacement of the functions $a_{c, v}^{+}\left(a_{c, v}\right)$ by the corresponding creation (annihilation) operators (below we omit the symbols ^ everywhere). The structure of the expansions (12) and (13) is defined by compatibility with the canonical anti-commutation relations

$$
\left\{a_{c, v}(\mathbf{p}), a_{c, v}^{\dagger}(\mathbf{q})\right\}=\delta_{\mathbf{p}, \mathbf{q}}
$$

and so on.

Substituting Eqs.(12) and (13) in the Eqs.(7)-(9), we obtain the total Hamiltonian and charge in the diagonal form (the quasiparticle representation [6])

$$
\begin{aligned}
H_{t o t} & =\frac{2}{\sqrt{V}} \sum_{\mathbf{p}}[\varepsilon(\mathbf{p})+\Delta / 2]\left\{a_{c}^{\dagger}(\mathbf{p}) a_{c}(\mathbf{p})+a_{v}^{\dagger}(-\mathbf{p}) a_{v}(-\mathbf{p})\right\}, \\
Q & =e \frac{2}{\sqrt{V}} \sum_{\mathbf{p}}\left\{a_{c}^{\dagger}(\mathbf{p}) a_{c}(\mathbf{p})-a_{v}^{\dagger}(-\mathbf{p}) a_{v}(-\mathbf{p})\right\},
\end{aligned}
$$

where the factor 2 is included here to account for the degeneracy with respect to the spin degrees of freedom. The present here energy degeneracy of the c-v- states is eliminated in the case $\epsilon_{c} \neq \epsilon_{v}$ (e.g., $m_{e} \neq m_{h}$ ). Thus, the decompositions (12), (13) and the quantization rules (14) lead to the well-known operator structure of macroscopic physical quantities.

The presented formalism allows to straightforwardly include a time- dependent electric field of arbitrary frequency and amplitude. Interaction with a quasiclassical electromagnetic field in the original coordinate representation is introduced by the substitution $\partial_{\mu} \rightarrow D_{\mu}=\partial_{\mu}+i e A_{\mu}(\mu=0,1,2,3)$, where $A_{\mu}^{e x}+A_{\mu}^{i n}$ is the 4-potential of the external and internal field. We will restrict ourselves below to the case of a nonstationary space-homogeneous electric field with 4-potential in the Hamilton gauge, $A_{\mu}=\left(0, A_{1}(t), A_{2}(t), A_{3}(t)\right)$ and then $\hat{\mathbf{p}} \rightarrow \hat{\mathbf{P}}=\hat{\mathbf{p}}+e \mathbf{A}$. The usual substitution into the Hamiltonian (7) leads to a non-diagonal form of the decompositions (12), (13) in the momentum representation. The adequate interpretation of the formalism is achieved by transition to quasiparticle representation, in which all observable operators have the diagonal form. Usually, the Bogoliubov method of time-dependent canonical transformations is used [6]. We will use below an economical method based on the holomorphic (oscillator) representation [15], that was developed in work [7] for the problem of the relativistic kinetics of vacuum pair creation in strong electromagnetic field.

In accordance with the method of work [7], it is necessary to make the substitution $\mathbf{p} \rightarrow \mathbf{P}$ in the dispersion law occurring in the decomposition (12) and (13) for the free field wave function and canonical momentum and also to introduce new time dependent amplitudes (or operators) $a_{c, v}(\mathbf{p}, t)$ by the replacement $a_{c, v}(\mathbf{p}) \exp [-i \varepsilon(\mathbf{p}) t] \rightarrow$ $a_{c, v}(\mathbf{p}, t)$ and so on. The result is the following:

$$
\begin{aligned}
& \Psi(\mathbf{x}, t)=\frac{1}{\sqrt{V}} \sum_{\mathbf{p}} \sqrt{\frac{\Delta}{\Delta+2 \varepsilon(\mathbf{P})}}\left\{a_{c}(\mathbf{p}, t)+a_{v}(-\mathbf{p}, t)\right\} e^{i \mathbf{p} \mathbf{x}}, \\
& \pi(\mathbf{x}, t)=\frac{i}{2 \sqrt{V}} \sum_{\mathbf{p}} \sqrt{\frac{\Delta+2 \varepsilon(\mathbf{P})}{\Delta}}\left\{a_{c}^{\dagger}(\mathbf{p}, t)-a_{v}^{\dagger}(-\mathbf{p}, t)\right\} e^{-i \mathbf{p x}} .
\end{aligned}
$$

Here it is assumed, that the dispersion law $\epsilon(\mathbf{p})$ is not changed under action of the external field besides the trivial displacement $\mathbf{p} \rightarrow \mathbf{P}$, i.e. the magnitude of the gap and the band boundaries remain invariable. Let us remark, that the Eqs. (17) and (18) allow the obvious generalization to the multiband model case. The total Hamiltonian $H_{t o t}$ can be obtained now from the free Hamiltonian (7) by the replacement $\partial \rightarrow D_{k}(\mathrm{k}=1,2,3)$. The subsequent substitution Eqs. (17), (18) leads at once to the diagonal form of the Hamiltonian in QP representation

$$
H_{t o t}(t)=\frac{2}{\sqrt{V}} \sum_{\mathbf{p}}[\varepsilon(\mathbf{P})+\Delta / 2]\left\{a_{c}^{\dagger}(\mathbf{p}, t) a_{c}(\mathbf{p}, t)+a_{v}^{\dagger}(-\mathbf{p}, t) a_{v}(-\mathbf{p}, t)\right\} .
$$

The new amplitudes $a_{e, h}(\mathbf{p}, t)$ obey the exact equations of motion, which can be obtained either from the minimal action principle or using the Hamilton equations $\dot{\Psi}=\delta H_{t o t} / \delta \pi(\mathbf{x}, t), \dot{\pi}=-\delta H_{t o t} / \delta \Psi(\mathbf{x}, t)$. Then 
the operator equations of motion follow after transition to the occupation number representation with the anticommutation relations

$$
\left\{a_{c, v}(\mathbf{p}, t), a_{c, v}^{\dagger}(\mathbf{q}, t)\right\}=\delta_{\mathbf{p q}}
$$

(the remaining elementary anti-commutators equal zero). The Heisenberg-like equations of motion are the following:

$$
\begin{aligned}
& \dot{a}_{c}(\mathbf{p}, t)=\lambda(\mathbf{p}, t) a_{v}(-\mathbf{p}, t)+i\left[H_{t o t}(t), a_{c}(\mathbf{p}, t)\right], \\
& \dot{a}_{v}(\mathbf{p}, t)=\lambda(\mathbf{p}, t) a_{c}(-\mathbf{p}, t)+i\left[H_{t o t}(t), a_{v}(\mathbf{p}, t)\right],
\end{aligned}
$$

where the amplitude of interband transitions is

$$
\lambda(\mathbf{p}, t)=\frac{\dot{\varepsilon}(\mathbf{P})}{\Delta+2 \varepsilon(\mathbf{P})}=-\frac{e \mathbf{P E}(t)}{m[\Delta+2 \varepsilon(\mathbf{P})]},
$$

and $E(t)=-\dot{A}(t)$ is the electric field strength. The first terms on r.h.s. of these equations describe the coupling of states related to different energy bands. Hence, the parameter (21) is the interband transition amplitude.

It is very important, that the Hamiltonian and the total charge operator have the diagonal form in this representation. Thus, the oscillator representation is simultaneously a quasiparticle one.

It is assumed that the electric field is switched off in the in - and out-states and the quasiparticle excitations become "free" and available for direct observation. In addition, it is also supposed here, that the system is found in the ground state at the initial moment $t_{0} \rightarrow-\infty$ and, hence, the initial state is the vacuum state $\mid 0>$ of electron and hole quasiparticles. This state is not equal to the out-state, where some quantity of electrons and holes can remain after switch off of the electric field.

\section{Kinetic equation and observables}

The basic object of the kinetic theory in the presence of an external strong field is the quasiparticle distribution function, which is defined on the in-vacuum state $\mid 0>$ in the present situation. In the case of a spacehomogeneous system, considered here, the quasiparticle distribution functions of electrons and holes are:

$$
f_{c, v}(\mathbf{p}, t)=<0\left|a_{c, v}^{\dagger}(\mathbf{p}, t) a_{c, v}(\mathbf{p}, t)\right| 0>.
$$

The general vacuum state here is a product of the electron vacuum states of the valence and conduction bands. Differentiating the functions (22) with respect to time, and using the equations of motion (20) we obtain:

$$
\dot{f}_{c, v}(\mathbf{p}, t)=\lambda(\mathbf{p}, t)\left\{f_{c, v}(\mathbf{p}, t)+f_{v, c}(\mathbf{p}, t)\right\},
$$

where the auxiliary correlation functions (the interband polarization functions) are introduced

$$
f_{c v}(\mathbf{p}, t)=<0\left|a_{c}^{\dagger}(\mathbf{p}, t) a_{v}(\mathbf{p}, t)\right| 0>, \quad f_{v c}(\mathbf{p}, t)=<0\left|a_{v}^{\dagger}(\mathbf{p}, t) a_{c}(\mathbf{p}, t)\right| 0>.
$$

The equation of motion for the functions (24) can be obtained by analogy with the equation (23). We can write it down in the integral form

$$
f_{c v}^{( \pm)}(\mathbf{p}, t)=\int_{-\infty}^{t} d t^{\prime} \lambda\left(\mathbf{p}, t^{\prime}\right)\left[1-f_{e}\left(\mathbf{p}, t^{\prime}\right)-f_{h}\left(-\mathbf{p}, t^{\prime}\right)\right] e^{ \pm 2 i \theta\left(\mathbf{p}, t, t^{\prime}\right)}
$$

where now $f_{c}=f_{e}$ and $f_{v}=1-f_{h}, f_{e}$ and $f_{h}$ are the electron and hole distribution functions. In Eq.(25) the following initial conditions have been introduced,

$$
\lim _{t \rightarrow-\infty} f_{c v}^{( \pm)}(\mathbf{p}, t)=0
$$

The resulting closed form of the KE now follows from Eqs. (23) and (24) [7]

$$
\dot{f}(\mathbf{p}, t)=2 \lambda(\mathbf{p}, t) \int_{-\infty}^{t} d t^{\prime} \lambda\left(\mathbf{p}, t^{\prime}\right)\left[1-2 f\left(\mathbf{p}, t^{\prime}\right)\right] \cos 2 \theta\left(\mathbf{p}, t, t^{\prime}\right) .
$$


This equation is equivalent to an integral equation of the Volterra type. The right-hand side of the KE (27) represents the source of creation and annihilation of electron-hole pairs and has the same form as in QED [8] (with an essential difference in construction of the amplitude $\lambda(\mathbf{p}, t)$ ), where the corresponding $\mathrm{KE}$ describe vacuum creation of electron-positron pairs. There is an other essential difference from QED kinetics, where $m$ is the unique mass parameter: in the present model there are two such parameters, $m$ and $\Delta$. That leads to some specific behavior of the e-h-system (see Sect. 4). In more realistic models must become apparent other details of energy spectrums of electrons and holes. Thus, this non-Markovian KE is a non-perturbative result in the mean-field approximation, within a quasiclassical treatment of the external and internal electric fields. KE (27) can be rewritten in the evident gauge invariant form if we make the change of variables $\mathbf{p} \rightarrow \mathbf{P}$ in the distribution functions $f(\mathbf{p}, t) \rightarrow f(\mathbf{P}, t)$.

As can be seen from the structure of KE (27) and amplitude (21), the interband transitions are caused by carrier acceleration in an electric field on the background of the changes of their energy (the denominator in Eq.(21)) and quantum beating with the alternating phase $\theta\left(\mathbf{p}, t, t^{\prime}\right)=\int_{t^{\prime}}^{t} d \tau[\varepsilon(\mathbf{P}(\tau)+\Delta / 2]$.

The KE (27) can be transformed to a system of ordinary differential equations, which is convenient for numerical analysis

$$
\dot{f}=\lambda u, \quad \dot{u}=2 \lambda(1-2 f)-(2 \varepsilon+\Delta) v, \quad \dot{v}=(2 \varepsilon+\Delta) u
$$

where $u+i v=2 f^{(+)}$and $f^{(+)}$is given by Eq. (25). These equations have the first integral $(1-2 f)^{2}+u^{2}+v^{2}=$ 1 , according to which the phase trajectories are located on an ellipsoid with top coordinates $f=u=v=0$ and $f=1, u=v=0$.

In the case of a small non-equilibrium correction to the initial distribution function, $\delta f(\vec{p}, t) \ll f_{0}(\vec{p}, t)$, we obtain the closed solution of Eq.(27)

$$
\begin{aligned}
& \delta f(\mathbf{p}, t)=\left[1-2 f_{0}(\mathbf{p})\right]\left|\int_{-\infty}^{t} d t^{\prime} \lambda\left(\mathbf{p}, t^{\prime}\right) e^{2 i \theta\left(\mathbf{p}, t, t^{\prime}\right)}\right|^{2}, \\
& u(\mathbf{p}, t)=2\left[1-2 f_{0}(\mathbf{p})\right] \int_{-\infty}^{t} d t^{\prime} \lambda\left(\mathbf{p}, t^{\prime}\right) \cos 2 \theta\left(\mathbf{p}, t, t^{\prime}\right) .
\end{aligned}
$$

After the distribution functions of electron and hole quasiparticles have been obtained, we can write down the densities of observables by averaging of the Hamilton operator (15), the electron charge (16), the total electronhole current etc. over the vacuum state. As a result, we have the time dependent densities of total energy $\omega(t)$, carrier number and total current in the form $\left([d \mathbf{p}]=(2 \pi)^{-3} d^{3} p\right)$ :

$$
\begin{aligned}
\omega(t) & =2 \int[d \mathbf{p}][2 \varepsilon(\mathbf{p}, t)+\Delta] f(\mathbf{p}, t), \\
n(t) & =4 \int[d \mathbf{p}] f(\mathbf{p}, t), \\
\mathbf{j}(t) & =\frac{4 e}{m} \int[d \mathbf{p}] \mathbf{p}\{f(\mathbf{p}, t)+u(\mathbf{p}, t)\}=\mathbf{j}_{\text {cond }}(t)+\mathbf{j}_{\text {pol }}(t),
\end{aligned}
$$

i.e. the total current is the sum of conductivity and polarization currents. The Eqs. (31)-(33) are written in the thermodynamical limit, $V \rightarrow \infty$. The integrations here are carried out over the first Brillouin zone, $p_{\max }=\pi / a$.

If the electric field is rather large $\left(|E(t)| \sim E_{c}\right.$ ), it is necessary to take into account the induced electric field $\mathbf{E}_{i n}(t)$ produced by the electron-hole plasma due to the external field $\mathbf{E}_{e x}(t)$. Thus, the total field is $\mathbf{E}_{t o t}(t)=$ $\mathbf{E}_{i n}(t)+\mathbf{E}_{e x}(t)$. The induced field $\mathbf{E}_{i n}(t)$ obeys the Maxwell equation with the current (33): $\dot{\mathbf{E}}_{i n}(t)=-4 \pi \mathbf{j}$. This equation together with the KE (27) (or the system (28)) forms the self-consistent nonlinear system of the back reaction problem. 


\section{Comparison with other approaches}

Equations (28) can be compared with the corresponding KE systems in the theory of the interband transitions in framework of other approaches. In all cases these KE's have the same mathematical structure but differ only by (momentum, electric field and so on) dependence of the coefficients in the functions $f, u, v$. The similarity of these KE's is stipulated by the circumstance that the complete description of the interband transitions in the framework of the mean field approximation is based in all these cases on the assignment of three correlators (22) and (24). The structure of the coefficients is defined in the first turn by selection of the method of coupling to the electric field: either the standard way based on the substitution $\vec{p} \rightarrow \vec{P}$ or the dipole approximation $H_{i n}=-e \vec{x} \vec{E}(t)$. Both approaches are equivalent in a space with a continuous translational symmetry group [16] which is proven by the gauge transformation of the wave function $\Psi(\mathbf{x}, t)=\exp \{-i \mathbf{A}(t) \mathbf{x}\} \tilde{\Psi}(\mathbf{x}, t)$. However, this transformation is impossible in a space with a discrete translational symmetry group. As a result, the two approaches may lead to different results. From this point of view, the KE's (28) are exact while the Bloch KE's [9] are approximate, independently of employment of the nonstationary perturbation theory.

The presented KE's (28) are close to the KE's obtained in the work [12] (see also the book [13]). However, in these works some additional approximations were used: the neglect of the $A^{2}$ term in the primordial Hamiltonian (importance of the retention of this term was underlined in the works [17]) and the resonance approximation $0<\nu-\Delta \ll \Delta$ ( $\nu$ is the frequency of the quasiclassical external electric field). Transition to the quasiclassical representation (in the work [12] for the Hamiltonian diagonalization was used the Bogoliubov method of the canonical transformations) unites both approximation.
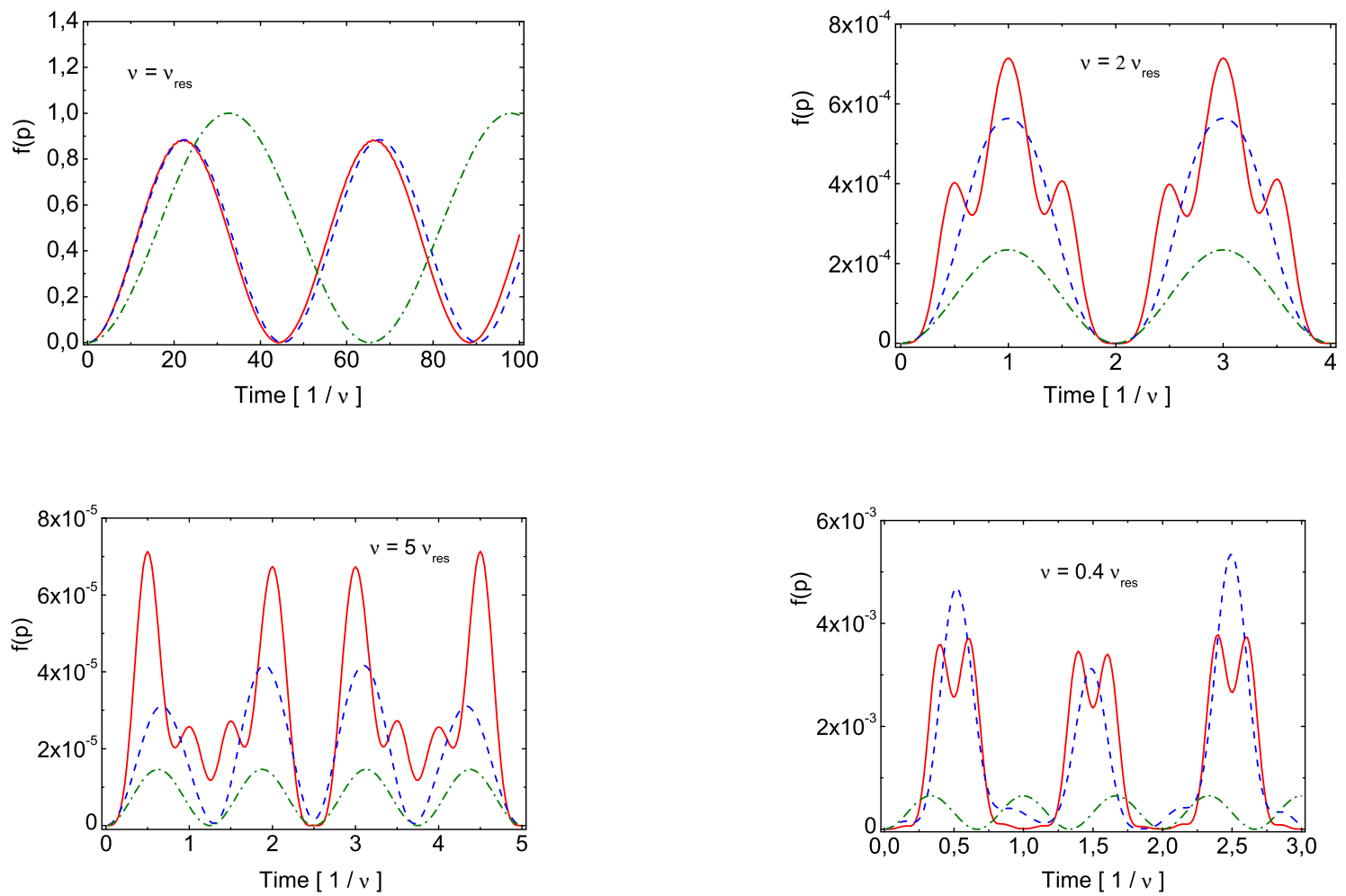

Fig. 1 Time dependence of $f(\mathbf{p}, t)$ in a monochromatic field with $E=10^{7} \mathrm{~V} / \mathrm{cm}$, energy gap $\Delta=1 \mathrm{eV}$ and effective mass $m=m_{e}$ in the vicinity of the "resonance" frequency $\nu_{\text {res }}=\Delta+\mathbf{p}^{2} / m$ at $|p|=8.6 \cdot 10^{-4} m_{e}(\lambda=600 \mathrm{~nm})$ : solid line - exact solution of Eqs. (28), dashed line - resonant approximation (34) and dashed-dotted line - solution of RWA equations (5.44) of Ref. [9]. 
Fig. 1 shows the comparison of the exact solutions of Eqs. (28) with different approximate solutions. The most resemblance is achieved in the framework of the rotating wave approximation (RWA) for a periodic signal [9]. In this case only the resonant terms are kept in the KE (27) (resonant approximation) which leads to the following system of equations (instead of Eqs. (28))

$$
\dot{f}_{R A}=\Lambda u_{R A}, \quad \dot{u}_{R A}=\frac{1}{2} \Lambda\left(1-2 f_{R A}\right)-(2 \varepsilon+\Delta-\nu) v_{R A}, \quad \dot{v}_{R A}=(2 \varepsilon+\Delta-\nu) u_{R A}
$$

where $\lambda=\Lambda(\vec{p}, t) \sin \nu t$. In particular, one can speak here about the analogue to Rabi flopping with a momentum and time dependent frequency $|\Lambda(\mathbf{p}, t)|$, see Fig. 1. The solution of Eqs.(28) at fixed momentum has a quasiresonant dependence on the frequency of the external field: at $\nu \approx \nu_{\text {res }}=\Delta+\mathbf{p}^{2} / \mathrm{m}$, the value of $f(\mathbf{p}, t)$ changes from 0 to 1 with "Rabi frequency" $\Lambda(\vec{p}, t)$. Also, nonlinear effects distinctive for KE's (28) are clearly visible in these pictures. Fig. 2a shows the time dependence of the created carrier density for a monochromatic field (this resembles the "accumulation” effect of vacuum pair creation [8].)
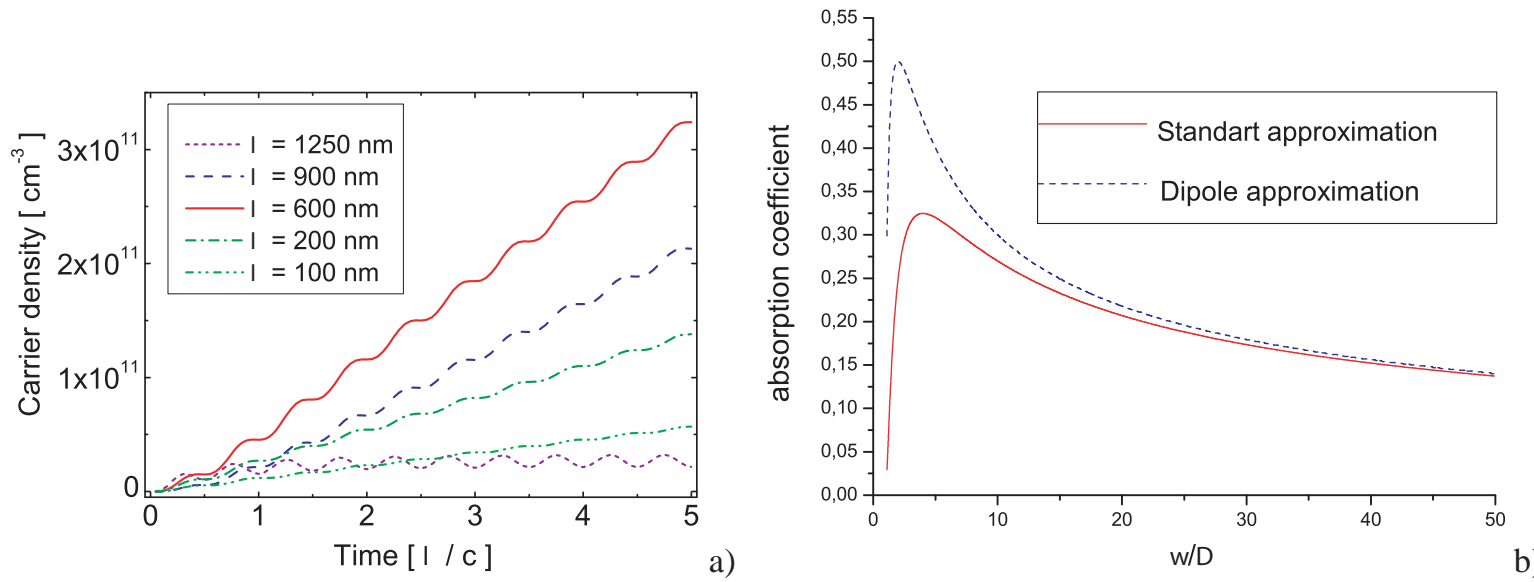

Fig. 2 Time dependence of created carrier density for the monochromatic field for $E=1000 \mathrm{~V} / \mathrm{cm}$ in the vicinity of the resonance frequency $\lambda=600 \mathrm{~nm}$, corresponding to the maximal pair creation rate- a); Absorption coefficient for $\mathrm{D}=3$ : solid line- the presented approach (Eq.(41)), dashed line- the dipole approximation (Eq.(42))- b).

The derivation procedure of the basic KE (27) shows (Sect. 3), that the limitation $m_{e}=m_{h}=m$ can be eliminated and one can instead understand $m$ as the effective mass of the carriers. The presented approach is valid for arbitrary physically reasonable parameters of a quasiclassical external electric field, in particular, for a harmonic field it is valid for the whole range of values of the Keldysh adiabatic parameter.

\section{Optical absorption}

Let us show that in the leading order of perturbation theory with respect to the parameter $E_{0} / E_{c} \ll 1$ ( $E_{0}$ is the amplitude of an external signal), the optical susceptibility is defined by the polarization part of the current (33) only. We consider the case that some free carriers are present in the initial state before switching on an external field.

In the Bloch approach, the optical susceptibility is defined by means of dipole moment density $\mathbf{P}(t)$. Below we will use a prescription which leads to connection of vector $\mathbf{P}(t)$ with the polarization function (30) in our approach. That allows to observe some close parallels and distinctions between both approaches. For a weak external field, comparison of the conductivity and polarization current densities (33) shows that we have the following estimations in the leading approximation in characteristic field strength $E$ :

$j_{\text {pol }}=j_{\text {pol }}^{(1)} \sim E, \quad j_{\text {cond }}=j_{\text {cond }}^{(2)} \sim E^{2}$, and hence according to Eq. (33) we have $\mathbf{j}(t) \cong \mathbf{j}_{\text {pol }}^{(1)}(t)=$ $\frac{4 e}{m} \int[d p] \mathbf{p} u^{(1)}(\mathbf{p}, t)$. On the other hand, one can use the general definition of the polarization current density 
and vector $\mathbf{P}(t)$

$$
\mathbf{j}_{\text {pol }}^{(1)}(t)=\dot{\mathbf{P}}(t), \quad \mathbf{P}(t)=\frac{4 e}{m} \int_{-\infty}^{t} d t^{\prime} \int[d p] \mathbf{p} u^{(1)}\left(\mathbf{p}, t^{\prime}\right) .
$$

Using the Eq. (33) for the electric field $\mathbf{E}(0,0, E(t))$ one can easily obtain for the Fourier component of the optical susceptibility [9]

$$
\xi(\omega)=\frac{4 e^{2}}{m_{r}^{2} \omega} \int[d p] \frac{\left(p^{3}\right)^{2}}{\Delta+2 \varepsilon^{(0)}}\left[1-2 f_{0}(\mathbf{p})\right] \times\left\{\frac{1}{\Delta+2 \varepsilon^{(0)}+\omega+i \delta}-\frac{1}{\Delta+2 \varepsilon^{(0)}-\omega+i \delta}\right\},
$$

where $f_{0}$ is the equilibrium distribution function of the free carriers and $\varepsilon^{(0)}=p^{2} / 2 m_{r}$ with the reduced electronhole mass $m_{r}$. The absorption coefficient $\alpha(\omega)=\operatorname{Im} \xi(\omega)$ is equal to

$$
\alpha(\omega)=\frac{4 \pi e^{2}}{m_{r}^{2}} \int[d p] \frac{\left(p^{3}\right)^{2}}{\left(\Delta+2 \varepsilon^{(0)}\right)^{2}}\left[1-2 f_{0}(\mathbf{p})\right] \delta\left(\Delta+2 \varepsilon^{(0)}-\omega\right) .
$$

Calculations for different dimensionalities lead to the following results:

$$
\begin{array}{rlrl}
D & =1: & \alpha(\omega) & =e^{2} \sqrt{\frac{\Delta}{m}} \frac{1}{\omega^{2}}\left(\frac{\omega}{\Delta}-1\right)^{1 / 2} F(\omega) \theta\left(\frac{\omega}{\Delta}-1\right), \\
D=2: & \alpha(\omega) & =\frac{e^{2} \Delta}{2} \frac{1}{\omega^{2}}\left(\frac{\omega}{\Delta}-1\right) F(\omega) \theta\left(\frac{\omega}{\Delta}-1\right), \\
D=3: & \alpha(\omega) & =\frac{e^{2} m^{1 / 2} \Delta^{3 / 2}}{3 \pi} \frac{1}{\omega^{2}}\left(\frac{\omega}{\Delta}-1\right)^{3 / 2} F(\omega) \theta\left(\frac{\omega}{\Delta}-1\right),
\end{array}
$$

where $F(\omega)$ is the statistical factor on the energy surface $P_{\omega}=\sqrt{m(\omega-\Delta)}$, i.e $F(\omega)=1-f_{0}\left(P_{\omega}\right)$. Thus, we obtain the characteristic frequency dependence for the different dimensions $D$ :

$$
\alpha_{D}(\omega) \sim \frac{1}{\omega^{2}}\left(\frac{\omega}{\Delta}-1\right)^{D / 2} .
$$

In the validity region of Eqs. (38)-(40) $\omega / \Delta \geq 1$ and $E / E_{c} \ll 1$, the Keldysh adiabatic parameter is large, $\gamma \gg 1$ corresponding to action of the multiphoton mechanism of absorption.

The standard theory of optical absorption based on the dipole approximation leads to a different frequency dependence [9]:

$$
\alpha_{D}^{d i p}(\omega) \sim \frac{1}{\omega^{2}}\left(\frac{\omega}{\Delta}-1\right)^{(D-2) / 2} .
$$

Technically, this circumstance is stipulated by the characteristic momentum dependence of the transition amplitude (21) in contrast to the Rabi frequency [9]. Fig.2b illustrates the features of the spectral behaviour of the absorption coefficient obtained in the framework of the dipole approximation Eq.(42) and on the basis of the presented approach Eq.(41) for $\mathrm{D}=3$.

\section{Summary}

We have presented a quantum kinetic theory of coherent e-h pair excitations exploiting the close analogy to vacuum pair creation in a strong field (dynamical Schwinger effect). For the mathematical realization of this idea, it is natural to construct (on the basis of Eq. (1)) an equation of motion of second order with respect to time, which combines the dispersion properties of both electron and hole. This equation was used for derivation of the KE for the description of coherent e-h excitations under action of a nonstationary electric field (dynamical Zener effect) obtained within a non-perturbative dynamical framework. The obtained KE was compared with KE's obtined in the frame of the Bloch equations. In the considered formalism, the frequency of the electric 
field is limited by condition of the quasiclassical field. It is obvious, that in the case of low frequency $\nu \lesssim \Delta$ (in the transparency region) the process of e-h excitation is essentially of multi-photon nature and stipulated by loss of stability of electron states in the valence band under action of an external field and, as a consequence, its transition to an excited state in the conduction band [18]. Let us summarize the main features of the proposed theory:

1. The given approach is a consecutive kinetic theory based on a non-perturbative dynamical foundation.

2. These KE's allow to describe states of carriers far from equilibrium under action of a time-dependent electric field. In particular, they allow to calculate the non-equilibrium distribution function and all relevant physical quantities.

3. The theory allows for various modifications and improvements on the dynamical level, such as inclusion of carrier-carrier interaction in the presence of a strong field as has been demonstrated for laser plasmas [19].

4. The presented theory is valid in the whole domain of the Keldysh adiabatic parameter and selfconsistently covers both - the tunnel and multiphoton excitation mechanisms.

The next important step on the way to a more adequate description of the non-stationary Zener effect is to take into account a realistic (non-parabolic) dispersion of electrons and holes. It will allow us to more adequately consider the region of strong fields which will be subject of future work.

Finally, the present results may be of interest also to vacuum pair creation. An experimental test of vacuum tunneling in QED requires enormous field strengths which are not available in the laboratory today. On the other hand, qualitatively similar results can be obtained in a much simpler manner by studying interband transitions in semiconductors with present day lasers.

Acknowledgements We thank Prof. V.P. Krainov for valuable discussions.

\section{References}

[1] C. Zener, Proc. Roy. Soc. Lond., 137, 696 (1932); J.R. Oppenheimer, Phys. Rev. 31, 66 (1928); L.V. Keldysh, Sov. Phys. JETP 33, 66 (1957).

[2] T. Meier, P. Thomas, and S.W. Koch, Coherent Semiconductor Optics, Springer, Berlin - Heidelberg - New York (2002).

[3] J. Rau and B. Muller, Phys. Rep. 272, 1 (1996).

[4] A.G. Green and S.L. Sondhi, Phys. Rev. Lett. 95, 267001 (2005); T. Oka and H. Aoki, Phys. Rev. Lett. 95, 137601 (2005); S.V. Morozov, K.S. Novoselov, M.I. Katsnelson, F. Schedin, D.C. Elias, J.A. Jaszczak, A.K. Geim, Phys. Rev. Lett. 100, 016602 (2008); L. A. Ponomarenko, F. Schedin, M. I. Katsnelson, R. Yang, E. H. Hill, K. S. Novoselov, A. K.Geim, Science 320, 356 (2008).

[5] J. Schwinger, Phys. Rev. 82, 664 (1951).

[6] A. Grib, S. Mamaev, and V. Mostepanenko, Vacuum quantum effects in strong external fields, Friedman Publisher company, St. Petersburg (1991).

[7] V. Pervushin, V. Skokov, A. Reichel, S. Smolyansky, and A. Prozorkevich, Int. J. Mod. Phys, A 20, 5689 (2005).

[8] C. D. Roberts, S. M. Schmidt, and D. V. Vinnik, Phys. Rev. Lett. 89, 153901 (2002).

[9] H. Haug and S.W. Koch, Quantum Theory of the Optic and Electronic Properties of Semiconductors, Fourth Ed., World Scientific (2004).

[10] M. Bonitz, Quantum Kinetic Theory, B.G. Teubner, Stuttgart-Leipzig (1998).

[11] N. Kwong, M. Bonitz, R. Binder, and H. Köhler, Phys. Stat. Sol. (b) 206, 197 (1998).

[12] V.M.Galitsky, S.P.Goreslavsky and V.F.Yelesin, Zh. Eksp. Teor. Fiz., 57, 207 (1969).

[13] V.M.Galitsky, V.F.Yelesin, Resonance Interaction of Electromagnetic Fields with Semiconductors, Atomizdat, Moscow (1986).

[14] A. Ringwald, Phys. Lett. B 510, 107 (2001).

[15] L. Faddeev and A. Slavnov, Gauge Fields: Introduction to Quantum Theory, Benjamin-Gummings, St. Peterburg (1984).

[16] N.B. Delone, V.P. Krainov, Atoms in Strong Light Fields. N.Y.: Springer Ser. Chem. Phys., Chap. 4, V. 28 (1985).

[17] H.R.Reiss, J. Opt. Soc. Am., B7, 574 (1990); Phys. Rev. A42, 1476 (1990).

[18] S.A. Smolyansky, A.V. Prozorkevich and M. Bonitz, Theor. Phys., Samara State University, 8, 95 (2007).

[19] D. Kremp, Th.Bornath, M. Bonitz, and M. Schlanges, Phys. Rev. E 60, 4725 (1999); M. Bonitz, Th. Bornath, D. Kremp, M. Schlanges, and W.D. Kraeft, Contrib. Plasma Phys. 39, 329 (1999); H. Haberland, M. Bonitz, and D. Kremp, Phys. Rev. E 64, 026405 (2001). 